\title{
Article
}

\section{A Variety of Nabla Hardy's Type Inequality on Time Scales}

\author{
Ahmed A. El-Deeb ${ }^{1, *}$, Samer D. Makharesh ${ }^{1}$, Sameh S. Askar ${ }^{2}$ (D) and Jan Awrejcewicz ${ }^{3, *(D)}$ \\ 1 Department of Mathematics, Faculty of Science, Al-Azhar University, Nasr City, Cairo 11884, Egypt \\ 2 Department of Statistics and Operations Research, College of Science, King Saud University, P.O. Box 2455, \\ Riyadh 11451, Saudi Arabia \\ 3 Department of Automation, Biomechanics and Mechatronics, Lodz University of Technology, \\ 1/15 Stefanowski St., 90-924 Lodz, Poland \\ * Correspondence: ahmedeldeeb@azhar.edu.eg (A.A.E.-D.); jan.awrejcewicz@p.lodz.pl (J.A.)
}

check for updates

Citation: El-Deeb, A.A.; Makharesh, S.D.; Askar, S.S.; Awrejcewicz, J. A Variety of Nabla Hardy's Type Inequality on Time Scales. Mathematics 2022, 10, 722. https:// doi.org/10.3390/math10050722 Academic Editors: Martin Bohner and Alberto Cabada

Received: 29 November 2021 Accepted: 15 February 2022 Published: 24 February 2022

Publisher's Note: MDPI stays neutral with regard to jurisdictional claims in published maps and institutional affiliations.

Copyright: (C) 2022 by the authors Licensee MDPI, Basel, Switzerland. This article is an open access article distributed under the terms and conditions of the Creative Commons Attribution (CC BY) license (https:// creativecommons.org/licenses/by/ $4.0 /)$.

\begin{abstract}
The primary goal of this research is to prove some new Hardy-type $\nabla$-conformable dynamic inequalities by employing product rule, integration by parts, chain rule and $(\gamma, a)$-nabla Hölder inequality on time scales. The inequalities proved here extend and generalize existing results in the literature. Further, in the case when $\gamma=1$, we obtain some well-known time scale inequalities due to Hardy inequalities. Many special cases of the proposed results are obtained and analyzed such as new conformable fractional $h$-sum inequalities, new conformable fractional $q$-sum inequalities and new classical conformable fractional integral inequalities.
\end{abstract}

Keywords: conformable derivative; time scales; Hardy's inequality; $(\gamma, a)$-nabla Hölder inequality on timescales

MSC: 26D15; 26E70

\section{Introduction}

Over several decades Hardy-type inequalities have been attracted many researchers and several refinements and extensions have been done to the previous results, we refer the reader to the works [1-4] and also the book [5].

Hardy [6] proved that.

Theorem 1. If a sequence $\{\varrho(\varsigma)\}_{\varsigma=1}^{\infty} \geq 0$ is real valued. If $p>1$, we get

$$
\sum_{\zeta=1}^{\infty} \frac{1}{\varsigma^{p}}\left(\sum_{l=1}^{\varsigma} \varrho(\iota)\right)^{p} \leq\left(\frac{p}{p-1}\right)^{p} \sum_{\zeta=1}^{\infty} \varrho^{p}(\varsigma)
$$

Hardy [7] proved the continuous version of (1).

Theorem 2. Assume $\eta \geq 0$ is continuous function on $[0, \infty)$. If $p>1$, we get

$$
\int_{0}^{\infty} \frac{1}{\pi^{p}}\left(\int_{0}^{\pi} \eta(\omega) d s\right)^{p} d \pi \leq\left(\frac{p}{p-1}\right)^{p} \int_{0}^{\infty} \eta^{p}(\pi) d \pi
$$

where $\left(\frac{p}{p-1}\right)^{p}$ in (2) is sharp.

Copson [8] proved variant discrete version of Hardy inequality.

Theorem 3. Let a sequence $\{\varrho(n)\}_{n=1}^{\infty} \geq 0$ be real valued nonnegative. For $1<p$, we have

$$
\sum_{n=1}^{\infty}\left(\sum_{j=n}^{\infty} \zeta(j)\right)^{p} \leq p^{p} \sum_{n=1}^{\infty}(n \zeta(n))^{p} .
$$


Renaud [9] proved the reverse versions of inequality (3).

Theorem 4. Assume $\{\zeta(n)\}_{n=1}^{\infty}$ is a nonnegative and nonincreasing sequence of real numbers. If $p>1$, we get

$$
\sum_{\zeta=1}^{\infty}\left(\sum_{l=\zeta}^{\infty} \zeta(\iota)\right)^{p} \geq \sum_{\zeta=1}^{\infty} \varsigma^{p} \zeta^{p}(\varsigma)
$$

Furthermore, he proved the following inequalities:

$$
\begin{gathered}
\int_{0}^{\infty}\left(\int_{\pi}^{\infty} \zeta(\omega) d \omega\right)^{p} d \pi \geq \int_{0}^{\infty} \pi^{p} \zeta^{p}(\pi) d \pi . \\
\int_{0}^{\infty} \frac{1}{\pi^{p}}\left(\int_{0}^{\pi} \zeta(\omega) d \omega\right)^{p} d \pi \geq \frac{p}{p-1} \int_{0}^{\infty} \zeta^{p}(\omega) d \omega .
\end{gathered}
$$

In [10-14] many authors have studied many new dynamic inequalities. Řehák [14] is the first author proved the version of Hardy inequality on time scales that unifies (1) and (2).

Theorem 5. Suppose the time scale $\mathbb{T}$, with $\mho \in C_{r d}\left([a, \infty)_{\mathbb{T}},[0, \infty)\right)$. If $p>1$, then

$$
\int_{a}^{\infty}\left(\frac{\int_{a}^{\sigma(\varrho)} \eta(\omega) \Delta \omega}{\sigma(\varrho)-a}\right)^{p} \Delta \varrho<\left(\frac{p}{p-1}\right)^{p} \int_{a}^{\infty} \eta^{p}(\varrho) \Delta \varrho
$$

unless $\eta \equiv 0$.

Furthermore, if $\mu(\varrho) / \varrho \rightarrow 0$ as $\varrho \rightarrow \infty$, then we get the sharp (7).

In [15] the authors extended (6) on genera time scales as follows: If $p>1$,

$$
\int_{0}^{\infty} \frac{1}{\wp^{p}}\left(\int_{0}^{\wp} \eta(\omega) \Delta \omega\right)^{p} \Delta \wp \geq \frac{p}{p-1} \int_{0}^{\infty} \eta^{p}(\wp) \Delta \wp .
$$

El-Deeb et al. [16] generalized (8) that unify (4) and (5). If $p \geq 1$ and $\gamma>1$, we get

$$
\int_{a}^{\infty} \frac{\tilde{\lambda}(\zeta) \breve{\Psi}^{p}(\zeta)}{\tilde{\Lambda} \hat{\gamma}(\zeta)} \Delta \zeta \geq \frac{p}{\hat{\gamma}-1} \int_{a}^{\infty} \tilde{\lambda}(\zeta) \tilde{\Lambda}^{p-\hat{\gamma}}(\zeta) \eta^{p}(\zeta) \Delta \zeta
$$

where

$$
\breve{\Psi}(\zeta)=\int_{a}^{\zeta} \tilde{\lambda}(\omega) \omega(\omega) \Delta \omega, \quad \text { and } \quad \tilde{\Lambda}(\zeta)=\int_{a}^{\zeta} \tilde{\lambda}(\omega) \Delta \omega
$$

Furthermore, in 2020, El-Deeb et al. [17] proved that if $\vartheta,\rceil_{2} \geq 0$ such that $\frac{w^{\Delta}(\wp)}{w(\wp)} \leq$ $\theta\left(\frac{G^{\Delta}(\wp)}{G^{\sigma}(\wp)}\right)$ and $\left.\frac{v^{\Delta}(\wp)}{v^{\sigma}(\wp)} \leq\right\urcorner_{2}\left(\frac{K^{\Delta}(\wp)}{K(\wp)}\right)$, where

$$
G(\wp)=\int_{a}^{\wp} g(\omega) \Delta \omega \text { with } G(\infty)=\infty \quad \text { and } \quad K(\wp)=\int_{a}^{\wp} r(\omega) \mho(\omega) \Delta \omega, \quad \wp \in[a, \infty)_{\mathbb{T}},
$$
with $\alpha>\theta+1$, and $p \geq 1$, then

$$
\begin{aligned}
& \int_{a}^{\infty} k^{\sigma}(\wp) v^{\sigma}(\wp) w(\wp) g(\wp)\left(G^{\sigma}(\wp)\right)^{-\alpha}\left(K^{\sigma}(\wp)\right)^{p} \Delta \wp \\
& \leq\left(\frac{p+\mathcal{T}_{2}}{\alpha-\theta-1}\right)^{p} \int_{a}^{\infty} \frac{k^{\sigma}(\wp) v^{\sigma}(\wp) w(\wp) r^{p}(\wp) \mho^{p}(\wp)\left(G^{\sigma}(\wp)\right)^{\alpha(p-1)}}{g^{p-1}(\wp) G^{p(\alpha-1)}(\wp)} \Delta \wp .
\end{aligned}
$$

In $[1,3-5]$, several authors proved many new inequalities. See also $[18,19]$. 
In [19], the authors proved:

$$
\int_{\omega}^{\infty} \frac{\lambda(\wp)}{\chi^{k-\alpha+1}(\wp)}\left(\Theta^{\sigma}(\wp)\right)^{h} \Delta_{\alpha} \wp \geqslant\left(\frac{h}{\alpha-m}\right)^{h} \int_{\omega}^{\infty} \lambda(\wp) \xi^{h}(\wp) \chi^{h-m+\alpha-1}(\wp) \Delta_{\alpha} \wp .
$$

Lemma 1 ([20], $(\gamma, a)$-nabla Hölder inequality on timescales). Let $d, b \in \mathbb{T}$ where $b>d$. If $\gamma \in(0,1]$ and $\eta, \xi: \mathbb{T} \longrightarrow \mathbb{R}$, then

$$
\int_{d}^{b}|\eta(\wp) \xi(\wp)| \nabla_{a}^{\gamma} \wp \leq\left(\int_{d}^{b}|\eta(\wp)|^{p} \nabla_{a}^{\gamma} \wp\right)^{1 / p}\left(\int_{d}^{b}|\xi(\wp)|^{q} \nabla_{a}^{\gamma} \wp\right)^{1 / q},
$$

where $p, q>1$ and $1 / p+1 / q=1$. This inequality is reversed if $0<p<1$ and if $p<0$, or $q<0$.

In this work, we prove and extend some new Hardy's inequality obtained in [15-17] to a standard time scale and set up numerous new sharpened types of fractional conformable $\nabla$-integral of order $\gamma \in(0,1]$ on time scales. As a new work, we generalize the inequalities presented in those papers. New discrete Hardy's inequalities maybe proved by our results via conformable fractional on time scales.

\section{Preliminaries}

In PhD thesis of S. Hilger initiated the theory of time scale. This subject has since gained tremendous attention among many mathematician. It combines the continuous and discrete branch of mathematics into one theory [21]. In [11,22], the authors stated all basic rules and definitions regarding to the calculus of time scales. Most of the fractional analysis concepts maybe found in [11,22-24]. Suppose $\sigma: \mathbb{T} \rightarrow \mathbb{T}$, is forward jump operator

$$
\sigma(\wp):=\inf \{\omega \in \mathbb{T}: \omega>\wp\}, \quad \wp \in \mathbb{T},
$$

$\mu(\wp):=\sigma(\wp)-\wp$ and is forward graininess function.

In [25], Bendouma et al. presenred a new version of the nabla fractional derivative on timescales. Given $\epsilon>0$, there is a $\delta$ - neighborhood $U_{\wp} \subset \mathbb{T}$ of $\wp, \delta>0$, such that

$$
\left|[\eta(\rho(\wp))-\eta(\omega)] \wp^{1-\alpha}-\wp \nabla, \alpha(\eta)(\wp)[\rho(\wp)-\omega]\right| \leq \varepsilon|\rho(\wp)-\omega|
$$

for all $\omega \in U_{\wp}$. We define the nabla fractional integral as

$$
\int \eta(\wp) \nabla_{\alpha} \wp=\int \eta(\wp) \wp^{\alpha-1} \nabla \wp .
$$

By using $\widehat{G}_{n}(\wp, \omega)$ for $\omega, \wp \in \mathbb{T}$, Rahmat et al. [23] presented nabla derivative conformable which generalizes the claim in [26].

Definition 1. Suppose $[\omega, \wp] \subset \mathbb{T}$ and $\omega<\wp . \widehat{G}_{n}: \mathbb{T} \times \mathbb{T} \longrightarrow \mathbb{R}^{+}, n \in \mathbb{N}_{0}$ is presented as the generalized time scale power function by

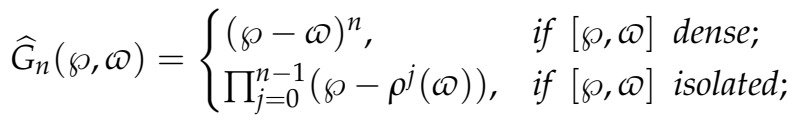

with inverse $\widehat{G}_{-n}: \mathbb{T} \times \mathbb{T} \longrightarrow \mathbb{R}^{+}$

$$
\widehat{G}_{-n}(\wp, \omega)= \begin{cases}(\wp-\omega)^{-n}, & \text { if }[\wp, \emptyset] \text { dense; } \\ \frac{1}{\prod_{j=0}^{n-1}\left(\rho^{n}(\wp)-\rho^{j}(\omega)\right)}, & \text { if }[\wp, \emptyset] \text { isolated } .\end{cases}
$$

We use the convention $\widehat{G}_{0}(\wp, ๗)=1$ for all $\omega, \wp \in \mathbb{T}$. 
Definition 2 (Conformable nabla derivative). Suppose $a \in \mathbb{T}, \mho$ is $\nabla$ differentiable at $\wp>a$, $\mho: \mathbb{T} \longrightarrow \mathbb{R}$ and let it be nable differentiable at $\wp$, and its $\nabla$ derivative is given by

$$
\nabla_{a}^{\gamma} \mho(\wp)=\widehat{G}_{1-\gamma}(\wp, a) \mho^{\nabla}(\wp) \quad \wp>a,
$$

since $\widehat{G}_{1-\gamma}(\wp, a)$ as defined in (12). Suppose $\nabla_{a}^{\gamma}[\mho(\wp)]$ exists in $(a, a+\epsilon)_{\mathbb{T}}, \epsilon>0$, then

$$
\nabla_{a}^{\gamma}[\mho(a)]=\lim _{\wp \longrightarrow a^{+}} \nabla_{a}^{\gamma}[\mho(\wp)]
$$

suppose the existence of $\lim _{\wp} \longrightarrow a^{+} \nabla_{a}^{\gamma}[\mho(\wp)]$.

We have $\zeta \xi: \mathbb{T} \longrightarrow \mathbb{R}$ is differentiable with

$$
\nabla_{a}^{\gamma}(\zeta \xi)=\left[\nabla_{a}^{\gamma} \zeta(\wp)\right] \xi(\wp)+\zeta^{\rho}(\wp)\left[\nabla_{a}^{\gamma} \xi(\wp)\right] .
$$

Lemma 2 (Integration by parts). Suppose that $d, b \in \mathbb{T}$ where $b>d$. If $\eta$, $\xi$ are conformable $(\gamma, a)$ - nabla fractional differentiable and $\gamma \in(0,1]$, then:

$$
\int_{d}^{b} \eta(\wp)\left[\nabla_{a}^{\gamma} \xi(\wp)\right] \nabla_{a}^{\gamma} \wp=[\eta(\wp) \xi(\wp)]_{d}^{b}-\int_{d}^{b}\left[\nabla_{a}^{\gamma} \eta(\wp)\right] \xi^{\rho}(\wp) \nabla_{a}^{\gamma} \wp .
$$

If $c \in[\rho(\tau), \tau]_{\mathbb{R}}$, we have

$$
\nabla_{a}^{\gamma}(\mathrm{Y} \circ \zeta)(t)=\mathrm{Y}^{\prime}(\zeta(c)) \nabla_{a}^{\gamma}(\zeta(t))
$$

Furthermore, we have

$$
\begin{aligned}
\nabla_{a}^{-\gamma} \mho(\wp) & =\int_{\wp_{1}}^{\wp_{2}} \mho(\tau) \nabla_{a}^{\gamma} \tau \\
& =\int_{\wp_{1}}^{\wp_{2}} \mho(\tau) \widehat{G}_{\gamma-1}\left(\sigma^{\gamma-1}(\tau), a\right) \nabla \tau,
\end{aligned}
$$

and for $\mathbb{T}=\mathbb{R}$

$$
\int_{a}^{\wp} \mho(\pi) \nabla_{a}^{\gamma} \pi=\int_{a}^{\wp} \mho(\pi)(\pi-a)^{\gamma-1} d \pi .
$$

With $h>0$, if $\mathbb{T}=h \mathbb{Z}$, we have $h$-sum given by

$$
\int_{a}^{\wp} \mho(\pi) \nabla_{a}^{\gamma} \pi=\sum_{\pi \in(a, \wp]} h \mho(\pi)\left(\rho^{\gamma-1}(\pi)-a\right)_{h}^{(\gamma-1)} .
$$

For $\mathbb{T}=q^{\mathbb{N}_{0}}$, we get

$$
\int_{a}^{\wp} \mho(\pi) \nabla_{a}^{\gamma} \pi=\sum_{\pi \in(a, \wp]} \pi(1-\tilde{q}) \mho(\pi)\left(\rho^{\gamma-1}(\pi)-a\right)_{\tilde{q}}^{(\gamma-1)} .
$$

\section{Main Results}

In this section, we assume that $\mathbb{T}$ is unbounded above.

Theorem 6. Let $\mathbb{T}$ be time scale for $m \in[0, \infty)_{\mathbb{T}}, \gamma \in(0,1]$ and $a>\wp$. Further, assume the $r d$-continuous functions $v, r, g, \mho, k, w \geq 0$, on $[m, \infty)_{\mathbb{T}}$ with $k$ is nondecreasing. Further, suppose we have $\vartheta, \neg_{2} \geq 0: \frac{\nabla_{a}^{\gamma} w(\wp)}{w(\wp)} \geqslant \vartheta\left(\frac{\nabla_{a}^{\gamma} G(\wp)}{G(\wp)}\right)$ and $\frac{\nabla_{a}^{\gamma} v(\wp)}{v^{\rho}(\wp)} \geqslant \neg_{2}\left(\frac{\nabla_{a}^{\gamma} K(\wp)}{K(\wp)}\right)$, where

$G(\wp)=\int_{m}^{\wp} g(\omega) \nabla_{a}^{\gamma} \omega$ with $G(\infty)=\infty$ and $K(\wp)=\int_{m}^{\wp} r(\omega) \mho(\omega) \nabla_{a}^{\gamma} \omega, \quad \wp \in[m, \infty)_{\mathbb{T}}$. If $0<p<\gamma$ and $\urcorner_{1}>\vartheta+1$, then 


$$
\begin{aligned}
& \int_{m}^{\infty} k^{\rho}(\wp) v^{\rho}(\wp) w(\wp) g(\wp)\left(G^{\rho}(\wp)\right)^{\gamma-\urcorner_{1}-1}\left(K^{\rho}(\wp)\right)^{p-\gamma+1} \nabla_{a}^{\gamma} \wp \\
& \geqslant\left(\frac{p+\urcorner_{2}-\gamma+1}{\urcorner_{1}-\vartheta-\gamma}\right)^{p} \int_{m}^{\infty} \frac{k^{\rho}(\wp) v^{\rho}(\wp) w(\wp) r^{p}(\wp) \mho^{p}(\wp)\left(G^{\rho}(\wp)\right)^{\left.(1-\gamma+\urcorner_{1}\right)(p-1)} K^{1-\gamma}(\wp)}{g^{p-1}(\wp) G^{\left.p(\urcorner_{1}-\gamma\right)}(\wp)} \nabla_{a}^{\gamma} \wp .
\end{aligned}
$$

Proof. Using (15) with

$$
\nabla_{a}^{\gamma} u(\wp)=w(\wp) g(\wp)\left(G^{\rho}(\wp)\right)^{\gamma-\urcorner_{1}-1} \quad z^{\rho}(\wp)=k^{\rho}(\wp) v^{\rho}(\wp)\left(K^{\rho}(\wp)\right)^{p-\gamma+1},
$$

we have

$$
\begin{aligned}
& \int_{m}^{\infty} k^{\rho}(\wp) v \rho(\wp) w(\wp) g(\wp)\left(G^{\rho}(\wp)\right)^{\gamma-T_{1}-1}\left(K^{\rho}(\wp)\right)^{p-\gamma+1} \nabla_{a}^{\gamma} \wp \\
& =\left[u(\wp) k(\wp) v(\wp) K^{p-\gamma+1}(\wp)\right]_{m}^{\infty}+\int_{m}^{\infty}(-u(\wp)) \nabla_{a}^{\gamma}\left(k(\wp) v(\wp) K^{p-\gamma+1}(\wp)\right) \nabla_{a}^{\gamma} \wp,
\end{aligned}
$$

where we assumed that

$$
u(\wp)=-\int_{\wp}^{\infty} w(\omega) g(\omega)\left(G^{\rho}(\omega)\right)^{\gamma-\urcorner_{1}-1} \nabla_{a}^{\gamma} \omega .
$$

Applying (16), (14), and the hypothesis $\frac{\nabla_{a}^{\gamma} w(\wp)}{w(\wp)} \geqslant \vartheta\left(\frac{\nabla_{a}^{\gamma} G(\wp)}{G^{\rho}(\wp)}\right)$, we have $c \in[\rho(๗), ๗]$ with

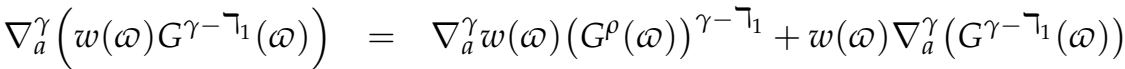

$$
\begin{aligned}
& \left.\geqslant \vartheta w(\omega) \nabla_{a}^{\gamma} G(\mathscr{\omega})\left(G^{\rho}(\mathscr{\omega})\right)^{\gamma-\urcorner_{1}-1}+(\gamma-\urcorner_{1}\right) w(\mathscr{\omega}) G^{\gamma-\urcorner_{1}-1}(c) \nabla_{a}^{\gamma} G(\mathscr{\omega}) \text {. }
\end{aligned}
$$

Since $\nabla_{a}^{\gamma} G(\omega)=g(\omega) \geq 0, c \geqslant \rho(\wp)$ and $\urcorner_{1}>\vartheta+\gamma$, we get

$$
\begin{aligned}
\nabla_{a}^{\gamma}\left(w(\omega) G^{\gamma-\urcorner_{1}}(\boldsymbol{\omega})\right) & \left.\geqslant \vartheta w(\omega) g(\omega)\left(G^{\rho}(\boldsymbol{\omega})\right)^{\gamma-\urcorner_{1}-1}+(\gamma-\urcorner_{1}\right) w(\omega) g(\boldsymbol{\omega})\left(G^{\rho}(\boldsymbol{\omega})\right)^{\gamma-\urcorner_{1}-1} \\
& \left.=(\gamma-\urcorner_{1}+\vartheta\right) w(\omega) g(\boldsymbol{\omega})\left(G^{\rho}(\boldsymbol{\omega})\right)^{\gamma-\urcorner_{1}-1} .
\end{aligned}
$$

This gives us that

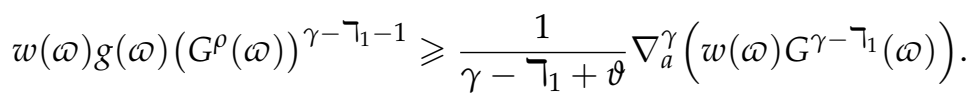

Hence

$$
\begin{aligned}
& -u(\wp)=\int_{\wp}^{\infty} w(\omega) g(\omega)\left(G^{\rho}(\omega)\right)^{\gamma-\urcorner_{1}-1} \nabla_{a}^{\gamma} \omega \geqslant \frac{1}{\gamma-\urcorner_{1}+\vartheta} \int_{\wp}^{\infty} \nabla_{a}^{\gamma}\left(w(\omega) G^{\gamma-\urcorner_{1}}(\omega)\right) \nabla_{a}^{\gamma} \omega \\
& =\frac{1}{\urcorner_{1}-\vartheta-\gamma} w(\wp) G^{\gamma-\urcorner_{1}}(\wp) \text {. }
\end{aligned}
$$

Applying (14) and (16), we have $c \in[\rho(\wp), \wp]$ with

$$
\begin{aligned}
\nabla_{a}^{\gamma}\left(k(\wp) v(\wp) K^{p-\gamma+1}(\wp)\right)= & \nabla_{a}^{\gamma}(k(\wp) v(\wp)) K^{p-\gamma+1}(\wp)+k^{\rho}(\wp) v^{\rho}(\wp) \nabla_{a}^{\gamma}\left(K^{p-\gamma+1}(\wp)\right) \\
= & \nabla_{a}^{\gamma} k(\wp) v(\wp) K^{p-\gamma+1}(\wp)+k^{\rho}(\wp) \nabla_{a}^{\gamma} v(\wp) K^{p-\gamma+1}(\wp) \\
& +(p-\gamma+1) k^{\rho}(\wp) v^{\rho}(\wp) K^{p-\gamma}(c) \nabla_{a}^{\gamma} K(\wp) .
\end{aligned}
$$




$$
\begin{aligned}
& \text { Since } \nabla_{a}^{\gamma} k(\wp) \geqslant 0, \nabla_{a}^{\gamma} K(\wp)=r(\wp) \mho(\wp) \geq 0, c \leq \wp, 0<p<\gamma \text { and } \frac{\nabla_{a}^{\gamma} v(\wp)}{v^{\rho}(\wp)} \geqslant \\
& \urcorner_{2}\left(\frac{\nabla_{a}^{\gamma} K(\wp)}{K(\wp)}\right) \text {, we have } \\
& \nabla_{a}^{\gamma}\left(k(\wp) v(\wp) K^{p-\gamma+1}(\wp)\right) \geqslant 7_{2} k^{\rho}(\wp) v^{\rho}(\wp) r(\wp) \mho(\wp) K^{p-\gamma}(\wp)+(p-\gamma+1) k^{\rho}(\wp) v^{\rho}(\wp) r(\wp) \mho(\wp) K^{p-\gamma}(\wp) \\
& \geqslant\left(p+7_{2}-\gamma+1\right) k^{\rho}(\wp) v^{\rho}(\wp) r(\wp) \mho(\wp) K^{p-\gamma}(\wp) \text {. } \\
& \int_{m}^{\infty} k^{\rho}(\wp) v^{\rho}(\wp) w(\wp) g(\wp)\left(G^{\rho}(\wp)\right)^{\gamma-\urcorner_{1}-1}\left(K^{\rho}(\wp)\right)^{p-\gamma+1} \nabla_{a}^{\gamma} \wp \\
& \geqslant \frac{p+\rceil_{2}-\gamma+1}{7_{1}-\vartheta-\gamma} \int_{m}^{\infty} k^{\rho}(\wp) v^{\rho}(\wp) w(\wp) r(\wp) \mho(\wp) G^{\gamma-\top_{1}}(\wp) K^{p-\gamma}(\wp) \nabla_{a}^{\gamma} \wp, \\
& \int_{m}^{\infty} k^{\rho}(\wp) v^{\rho}(\wp) w(\wp) g(\wp)\left(G^{\rho}(\wp)\right)^{\gamma-\urcorner_{1}-1}\left(K^{\rho}(\wp)\right)^{p-\gamma+1} \nabla_{a}^{\gamma} \wp \\
& \geqslant \frac{p+\rceil_{2}-\gamma+1}{\rceil_{1}-\vartheta-\gamma} \int_{m}^{\infty}\left(\left(k^{\rho}(\wp) v^{\rho}(\wp) w(\wp) g(\wp)\right)^{(p-1) / p}\left(G^{\rho}(\wp)\right)^{\left.(\gamma-\urcorner_{1}-1\right)(p-1) / p} K^{(p-1)(p-\gamma+1) / p}(\wp)\right) \\
& \times\left(\frac{\left(k^{\rho}(\wp) v^{\rho}(\wp) w(\wp)\right)^{1 / p} r(\wp) \mho \zeta(\wp)\left(G^{\rho}(\wp)\right)^{\left.(1-\gamma+\urcorner_{1}\right)(p-1) / p} K^{(1-\gamma) / p}(\wp)}{g^{(p-1) / p}(\wp) G^{\urcorner_{1}-\gamma(\wp)}}\right) \nabla_{a}^{\gamma} \wp .
\end{aligned}
$$

Applying (11) with index $p$ and index $p /(p-1)$, we obtain

$$
\begin{aligned}
& \int_{a}^{\infty} k^{\rho}(\wp) v^{\rho}(\wp) w(\wp) g(\wp)\left(G^{\rho}(\wp)\right)^{\left.(\gamma-\urcorner_{1}-1\right)}\left(K^{\rho}(\wp)\right)^{(p-\gamma+1)} \nabla_{a}^{\gamma} \wp \\
& \geqslant \frac{p+\rceil_{2}-\gamma+1}{\rceil_{1}-\vartheta-\gamma}\left(\int_{a}^{\infty} k^{\rho}(\wp) v^{\rho}(\wp) w(\wp) g(\wp)\left(G^{\rho}(\wp)\right)^{\left.(\gamma-\urcorner_{1}-1\right)} K^{(p-\gamma+1)}(\wp) \nabla_{a}^{\gamma} \wp\right)^{(p-1) / p} \\
& \times\left(\int_{a}^{\infty} \frac{k^{\rho}(\wp) v^{\rho}(\wp) w(\wp) r^{p}(\wp) \mho^{p}(\wp)\left(G^{\rho}(\wp)\right)^{\left.(1+\urcorner_{1}-\gamma\right)(p-1)} K^{1-\gamma}(\wp)}{g^{p-1}(\wp) G^{\left.p(\urcorner_{1}-\gamma\right)}(\wp)} \nabla_{a}^{\gamma} \wp\right)^{1 / p},
\end{aligned}
$$

which implies that

$$
\begin{aligned}
& \int_{m}^{\infty} k^{\rho}(\wp) v^{\rho}(\wp) w(\wp) g(\wp)\left(G^{\rho}(\wp)\right)^{\gamma-\urcorner_{1}-1}\left(K^{\rho}(\wp)\right)^{p-\gamma+1} \nabla_{a}^{\gamma} \wp \\
& \geqslant\left(\frac{p+\urcorner_{2}-\gamma+1}{\urcorner_{1}-\vartheta-\gamma}\right)^{p} \int_{m}^{\infty} \frac{k^{\rho}(\wp) v^{\rho}(\wp) w(\wp) r^{p}(\wp) \mho \mho^{p}(\wp)\left(G^{\rho}(\wp)\right)^{\left.(1-\gamma+\urcorner_{1}\right)(p-1)} K^{1-\gamma}(\wp)}{g^{p-1}(\wp) G^{\left.p(\urcorner_{1}-\gamma\right)}(\wp)} \nabla_{a}^{\gamma} \wp .
\end{aligned}
$$

We get our claim.

Remark 1. If one put $\gamma=1$ in Theorem 6, then we get the following inequality

$$
\begin{aligned}
& \int_{m}^{\infty} k^{\rho}(\wp) v^{\rho}(\wp) w(\wp) g(\wp)\left(G^{\rho}(\wp)\right)^{-\urcorner_{1}}\left(K^{\rho}(\wp)\right)^{p} \nabla \wp \\
& \geqslant\left(\frac{p+\urcorner_{2}}{7_{1}-\vartheta-1}\right)^{p} \int_{m}^{\infty} \frac{k^{\rho}(\wp) v^{\rho}(\wp) w(\wp) r^{p}(\wp) \mho^{p}(\wp)\left(G^{\rho}(\wp)\right)^{\urcorner_{1}(p-1)}}{g^{p-1}(\wp) G^{\left.p(\urcorner_{1}-1\right)}(\wp)} \nabla \wp .
\end{aligned}
$$

Corollary 1. When one put $\mathbb{T}=\mathbb{R}$ in (21) we get 


$$
\begin{aligned}
& \int_{m}^{\infty} k(\wp) v(\wp) w(\wp) g(\wp) G^{\gamma-\urcorner_{1}-1}(\wp) K^{p-\gamma+1}(\wp)(\wp-a)^{\gamma-1} d t \\
& \geqslant\left(\frac{p+\rceil_{2}-\gamma+1}{\rceil_{1}-\vartheta-\gamma}\right)^{p} \int_{m}^{\infty} \frac{k(\wp) v(\wp) w(\wp) r^{p}(\wp) \mho^{p}(\wp) G^{p-\rceil_{1}+\gamma-1}(\wp) K^{1-\gamma}(\wp)}{g^{p-1}(\wp)}(\wp-a)^{\gamma-1} d t . \\
& G(\wp)=\int_{m}^{\wp} g(\omega)(\omega-a)^{\gamma-1} d \omega \text { with } G(\infty)=\infty \text { and } K(\wp)=\int_{m}^{\wp} r(\omega) \mho(\omega)(\omega-a)^{\gamma-1} d \omega \text {. }
\end{aligned}
$$

Corollary 2. For $\mathbb{T}=h \mathbb{Z}, h>0$ in (21) we obtain

$$
\begin{aligned}
& \sum_{\wp=m}^{\infty} k(\wp-h) v(\wp-h) w(\wp) g(\wp) G^{\gamma-\urcorner_{1}-1}(\wp-h) K^{p-\gamma+1}(\wp-h)\left(\rho^{\gamma-1}(\wp)-a\right)_{h}^{(\gamma-1)} \\
& \geqslant\left(\frac{p+\rceil_{2}-\gamma+1}{\urcorner_{1}-\vartheta-\gamma}\right)^{p} \sum_{\wp=m}^{\infty} \frac{k(\wp-h) v(\wp-h) w(\wp) r^{p}(\wp) \mho^{p}(\wp)(G(\wp-h))^{\left.(1-\gamma+\urcorner_{1}\right)(p-1)} K^{1-\gamma}(\wp)\left(\rho^{\gamma-1}(\wp)-a\right)_{h}^{(\gamma-1)}}{g^{p-1}(\wp) G^{\left.p(\urcorner_{1}-\gamma\right)}(\wp)} .
\end{aligned}
$$

where

$$
G(\wp)=h \sum_{\omega=m}^{\wp} g(\omega)\left(\rho^{\gamma-1}(\omega)-a\right)_{h}^{(\gamma-1)} \text { with } \quad G(\infty)=\infty \quad \text { and } \quad K(\wp)=h \sum_{\omega=m}^{\wp} r(\omega) \mho(\omega)\left(\rho^{\gamma-1}(\infty)-a\right)_{h}^{(\gamma-1)} \text {. }
$$

Corollary 3. If $\mathbb{T}=\mathbb{Z}$, with $h=1$ in our Corollary 16 we have from (21) that

$$
\begin{aligned}
& \sum_{\wp=m}^{\infty} k(\wp-1) v(\wp-1) w(\wp) g(\wp) G^{\gamma-\urcorner_{1}-1}(\wp-1) K^{p-\gamma+1}(\wp-1)\left(\rho^{\gamma-1}(\wp)-a\right)^{(\gamma-1)} \\
& \geqslant\left(\frac{p+\rceil_{2}-\gamma+1}{\rceil_{1}-\vartheta-\gamma}\right)^{p} \sum_{\wp=m}^{\infty} \frac{k(\wp-1) v(\wp-1) w(\wp) r^{p}(\wp) \mho^{p}(\wp)(G(\wp-1))^{\left.(1-\gamma+\rceil_{1}\right)(p-1)} K^{1-\gamma}(\wp)\left(\rho^{\gamma-1}(\wp)-a\right)^{(\gamma-1)}}{g^{p-1}(\wp) G^{\left.p(\urcorner_{1}-\gamma\right)}(\wp)} . \\
& G(\wp)=h \sum_{\omega=m}^{\wp} g(\omega)\left(\rho^{\gamma-1}(\omega)-a\right)^{(\gamma-1)} \quad \text { with } \quad G(\infty)=\infty \quad \text { and } \quad K(\wp)=h \sum_{\omega=m}^{\wp} r(\omega) \mho(\omega)\left(\rho^{\gamma-1}(\omega)-a\right)^{(\gamma-1)} .
\end{aligned}
$$

Corollary 4. For $\mathbb{T}=q^{\mathbb{N}_{0}}$, in (21) we obtain

$$
\begin{aligned}
& \sum_{\wp=m}^{\infty} \wp\left(\rho^{\gamma-1}(\wp)-a\right)_{\tilde{q}}^{(\gamma-1)} k^{\rho}(\wp) v^{\rho}(\wp) w(\wp) g(\wp)\left(G^{\rho}(\wp)\right)^{\gamma-\urcorner_{1}-1}\left(K^{\rho}(\wp)\right)^{p-\gamma+1} \\
& \geqslant\left(\frac{p+\rceil_{2}-\gamma+1}{\rceil_{1}-\vartheta-\gamma}\right)^{p} \sum_{\wp=m}^{\infty} \frac{\wp\left(\rho^{\gamma-1}(\wp)-a\right)_{\tilde{q}}^{(\gamma-1)} k^{\rho}(\wp) v^{\rho}(\wp) w(\wp) r^{p}(\wp) \mho^{p}(\wp)\left(G^{\rho}(\wp)\right)^{\left.(1-\gamma+\urcorner_{1}\right)(p-1)} K^{1-\gamma}(\wp)}{g^{p-1}(\wp) G^{\left.p(\urcorner_{1}-\gamma\right)}(\wp)} .
\end{aligned}
$$

Theorem 7. Suppose $\mathbb{T}$ is a time scale with $m \in[0, \infty)_{\mathbb{T}}, \gamma \in(0,1]$ and $a>\wp$. Furthermore, suppose the $r d$-continuous functions $w, k, \mho, g, r, v \geq 0$ on $[m, \infty)_{\mathbb{T}}$ with $k$ nonincreasing. Furthermore, assume we have $\vartheta,\urcorner_{2} \geq 0$ with $\frac{\nabla_{a}^{\gamma} w(\wp)}{w^{\rho}(\wp)} \leqslant \vartheta\left(\frac{\nabla_{a}^{\gamma} G(\wp)}{G(\wp)}\right)$ and $\frac{\nabla_{a}^{\gamma} v(\wp)}{v(\wp)} \leqslant$ \urcorner$_{2}\left(\frac{\nabla_{a}^{\gamma} \mho(\wp)}{\mho^{\rho}(\wp)}\right)$, where

$$
G(\wp)=\int_{m}^{\wp} g(\omega) \nabla_{a}^{\gamma} \omega \text { with } G(\infty)=\infty, \quad \text { and } \quad \mho(\wp)=\int_{\wp}^{\infty} r(\omega) \mho(\omega) \nabla_{a}^{\gamma} \omega, \quad \wp \in[m, \infty)_{\mathbb{T}} .
$$
If $0<p<\gamma$ and $0 \leq\urcorner_{1}<\gamma$, then 


$$
\begin{aligned}
& \int_{m}^{\infty} k(\wp) v(\wp) w^{\rho}(\wp) g(\wp)\left(G^{\rho}(\wp)\right)^{\gamma-\urcorner_{1}+1} \mho^{p-\gamma+1}(\wp) \nabla_{a}^{\gamma} \wp \\
& \geqslant\left(\frac{p+\rceil_{2}-\gamma+1}{\gamma-\rceil_{1}+\vartheta}\right)^{p} \int_{m}^{\infty} \frac{k(\wp) v(\wp) w^{\rho}(\wp) r^{p}(\wp) \mho^{p}(\wp)\left(G^{\rho}(\wp)\right)^{p-\rceil_{1}+\gamma-1}\left(\mho^{\rho}(\wp)\right)^{1-\gamma}}{g^{p-1}(\wp)} \nabla_{a}^{\gamma} \wp .
\end{aligned}
$$

Proof. Applying (15), we get

$$
\begin{aligned}
& \int_{m}^{\infty} k(\wp) v(\wp) w^{\rho}(\wp) g(\wp)\left(G^{\rho}(\wp)\right)^{\gamma-\urcorner_{1}-1} \mho^{p-\gamma+1}(\wp) \nabla_{a}^{\gamma} \wp \\
& =\left[u(\wp) k(\wp) v(\wp) \mho^{p-\gamma+1}(\wp)\right]_{a}^{\infty}+\int_{m}^{\infty} u^{\rho}(\wp) \nabla_{a}^{\gamma}\left(-k(\wp) v(\wp) \mho^{p-\gamma+1}(\wp)\right) \nabla_{a}^{\gamma} \wp,
\end{aligned}
$$

where

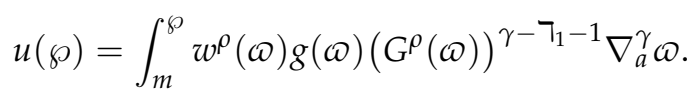

Applying(16), (14), and the assumption $\frac{\nabla_{a}^{\gamma} w(\wp)}{w^{\rho}(\wp)} \leqslant \vartheta\left(\frac{\nabla_{a}^{\gamma} G(\wp)}{G(\wp)}\right)$, we have $c \in[\rho(\omega), \omega]$ with

$$
\begin{aligned}
\nabla_{a}^{\gamma}\left(w(\omega) G^{\gamma-\urcorner_{1}}(\boldsymbol{\omega})\right) & =\nabla_{a}^{\gamma} w(\omega) G^{\gamma-\urcorner_{1}}(\boldsymbol{\omega})+w^{\rho}(\omega) \nabla_{a}^{\gamma}\left(G^{\gamma-\urcorner_{1}}(\boldsymbol{\omega})\right) \\
& \left.\leqslant \vartheta w^{\rho}(\boldsymbol{\omega}) G^{\gamma-\urcorner_{1}-1}(\omega) \nabla_{a}^{\gamma} G(\omega)+(\gamma-\urcorner_{1}\right) w^{\rho}(\boldsymbol{\omega}) G^{\gamma-\urcorner_{1}-1}(c) \nabla_{a}^{\gamma} G(\omega) .
\end{aligned}
$$

As $\nabla_{a}^{\gamma} G(\omega)=g(\omega) \geq 0, c \geqslant \rho(\omega)$ and $\left.0 \leq\right\urcorner_{1}<\gamma$, we get

$$
\begin{aligned}
\nabla_{a}^{\gamma}\left(w(\omega) G^{\gamma-\urcorner_{1}}(\boldsymbol{\omega})\right) & \left.\leqslant \vartheta w^{\rho}(\boldsymbol{\omega}) g(\omega)\left(G^{\rho}(\boldsymbol{\omega})\right)^{\gamma-\urcorner_{1}-1}+(\gamma-\urcorner_{1}\right) w^{\rho}(\boldsymbol{\omega}) g(\omega)\left(G^{\rho}(\boldsymbol{\omega})\right)^{\gamma-\urcorner_{1}-1} \\
& \left.=(\gamma-\urcorner_{1}+\vartheta\right) w^{\rho}(\boldsymbol{\omega}) g(\boldsymbol{\omega})\left(G^{\rho}(\boldsymbol{\omega})\right)^{\gamma-\urcorner_{1}-1},
\end{aligned}
$$

which implies

$$
w^{\rho}(\mathfrak{\omega}) g(\mathfrak{\omega})\left(G^{\rho}(\mathfrak{\omega})\right)^{\gamma-\urcorner_{1}-1} \geqslant \frac{1}{\gamma-\urcorner_{1}+\vartheta} \nabla_{a}^{\gamma}\left(w(\mathfrak{\omega}) G^{\gamma-\urcorner_{1}}(\mathfrak{\omega})\right) .
$$

Therefore

$$
\begin{aligned}
u^{\rho}(\wp)=\int_{m}^{\rho(\wp)} w^{\rho}(\omega) g(\omega)\left(G^{\rho}(\omega)\right)^{\gamma-\urcorner_{1}-1} \nabla_{a}^{\gamma} \omega & \geqslant \frac{1}{\gamma-\urcorner_{1}+\vartheta} \int_{m}^{\rho(\wp)} \nabla_{a}^{\gamma}\left(w(\omega) G^{\gamma-\urcorner_{1}}(\omega)\right) \nabla_{a}^{\gamma} \omega \\
& =\frac{1}{\gamma-\urcorner_{1}+\vartheta} w^{\rho}(\wp)\left(G^{\rho}(\wp)\right)^{\gamma-\urcorner_{1}} .
\end{aligned}
$$

Utilizing (14) and (16), we have $c \in[\rho(\wp), \wp]$ with

$$
\begin{aligned}
\nabla_{a}^{\gamma}\left(-k(\wp) v(\wp) \mho^{p-\rceil_{1}+1}(\wp)\right)= & -\left(\nabla_{a}^{\gamma}(k(\wp) v(\wp))\left(\mho^{\rho}(\wp)\right)^{p-\rceil_{1}+1}+k(\wp) v(\wp) \nabla_{a}^{\gamma}\left(\mho^{p-\gamma+1}(\wp)\right)\right) \\
= & -\left(\nabla_{a}^{\gamma} k(\wp) v^{\rho}(\wp)\left(\mho^{\rho}(\wp)\right)^{p-\gamma+1}+k(\wp) \nabla_{a}^{\gamma} v(\wp)\left(\mho^{\rho}(\wp)\right)^{p-\gamma+1}\right. \\
& \left.+(p-\gamma+1) k(\wp) v(\wp) \mho^{p-\gamma}(c) \nabla_{a}^{\gamma} \mho(\wp)\right) .
\end{aligned}
$$

Since $\nabla_{a}^{\gamma} k(\wp) \leqslant 0, \nabla_{a}^{\gamma} \mho(\wp)=-r(\wp) \mho(\wp) \leq 0, c \geqslant \rho(\wp), 0<p<\gamma$ and $\frac{\nabla_{a}^{\gamma} v(\wp)}{v(\wp)} \leqslant$ \urcorner$_{2}\left(\frac{\nabla_{a}^{\gamma} \mho(\wp)}{\mho^{\rho}(\wp)}\right)$, we get

$$
\begin{aligned}
\nabla_{a}^{\gamma}\left(-k(\wp) v(\wp) \mho^{p-\gamma+1}(\wp)\right) & \geqslant\rceil_{2} k(\wp) v(\wp) r(\wp) \mho(\wp)\left(\mho^{\rho}(\wp)\right)^{p-\gamma}+(p-\gamma+1) k(\wp) v(\wp) r(\wp) \mho(\wp) \mho^{p-\gamma}(\wp) \\
& \left.\geqslant(p-\gamma+\rceil_{2}+1\right) k(\wp) v(\wp) r(\wp) \mho(\wp)\left(\mho^{\rho}(\wp)\right)^{p-\gamma} .
\end{aligned}
$$


From (25)-(27), with $\mho(\infty)=0$ and $u(m)=0$ we obtain

$$
\begin{aligned}
& \int_{m}^{\infty} k(\wp) v(\wp) w^{\rho}(\wp) g(\wp)\left(G^{\rho}(\wp)\right)^{\gamma-\urcorner_{1}-1} \mho^{p-\gamma+1}(\wp) \nabla_{a}^{\gamma} \wp \\
& \geqslant \frac{p-\gamma+\urcorner_{2}+1}{\gamma-\urcorner_{1}+\vartheta} \int_{m}^{\infty} k(\wp) v(\wp) w^{\rho}(\wp) r(\wp) \mho(\wp)\left(G^{\rho}(\wp)\right)^{\gamma-\urcorner_{1}}\left(\mho^{\rho}(\wp)\right)^{p-\gamma} \nabla_{a}^{\gamma} \wp,
\end{aligned}
$$

which is equivalent to

$$
\begin{aligned}
& \int_{m}^{\infty} k(\wp) v(\wp) w^{\rho}(\wp) g(\wp)\left(G^{\rho}(\wp)\right)^{\gamma-\urcorner_{1}-1} \mho^{p-\gamma+1}(\wp) \nabla_{a}^{\gamma} \wp \\
& \geqslant \frac{p+\rceil_{2}-\gamma+1}{\gamma-\urcorner_{1}+\vartheta} \int_{m}^{\infty}\left(\left(k(\wp) v(\wp) w^{\rho}(\wp) g(\wp)\right)^{(p-1) / p}\left(G^{\rho}(\wp)\right)^{\left.(\gamma-\rceil_{1}-1\right)(p-1) / p}\left(\mho^{\rho}(\wp)\right)^{(p-1)(p-\gamma+1) / p}\right) \\
& \quad \times\left(\frac{\left(k(\wp) v(\wp) w^{\rho}(\wp)\right)^{1 / p} r(\wp) \mho(\wp)\left(G^{\rho}(\wp)\right)^{\left.(p-\rceil_{1}+\gamma-1\right) / p}\left(\mho^{\rho}(\wp)\right)^{(1-\gamma) / p}}{g^{(p-1) / p}(\wp)}\right) \nabla_{a}^{\gamma} \wp .
\end{aligned}
$$

Applying (11) with the index $p$ and the index $p /(p-1)$, obtains

$$
\begin{aligned}
& \int_{m}^{\infty} k(\wp) v(\wp) w^{\rho}(\wp) g(\wp)\left(G^{\rho}(\wp)\right)^{\gamma-\urcorner_{1}+1} \mho^{p-\gamma+1}(\wp) \nabla_{a}^{\gamma} \wp \\
& \geqslant \frac{p+\rceil_{2}-\gamma+1}{\gamma-\urcorner_{1}+\vartheta}\left(\int_{m}^{\infty} k(\wp) v(\wp) w^{\rho}(\wp) g(\wp)\left(G^{\rho}(\wp)\right)^{\gamma-\urcorner_{1}-1}\left(\mho^{\rho}(\wp)\right)^{p-\gamma+1} \nabla_{a}^{\gamma} \wp\right)^{(p-1) / p} \\
& \times\left(\int_{m}^{\infty} \frac{k(\wp) v(\wp) w^{\rho}(\wp) r^{p}(\wp) \mho^{p}(\wp)\left(G^{\rho}(\wp)\right)^{p-\urcorner_{1}+\gamma-1}\left(\mho^{\rho}(\wp)\right)^{1-\gamma}}{g^{p-1}(\wp)} \nabla_{a}^{\gamma} \wp\right)^{1 / p} .
\end{aligned}
$$

This leads to

$$
\begin{aligned}
& \int_{m}^{\infty} k(\wp) v(\wp) w^{\rho}(\wp) g(\wp)\left(G^{\rho}(\wp)\right)^{\gamma-\urcorner_{1}+1} \mho^{p-\gamma+1}(\wp) \nabla_{a}^{\gamma} \wp \\
& \geqslant\left(\frac{p+\rceil_{2}-\gamma+1}{\gamma-\rceil_{1}+\vartheta}\right)^{p} \int_{m}^{\infty} \frac{k(\wp) v(\wp) w^{\rho}(\wp) r^{p}(\wp) \mho^{p}(\wp)\left(G^{\rho}(\wp)\right)^{p-\urcorner_{1}+\gamma-1}\left(\mho^{\rho}(\wp)\right)^{1-\gamma}}{g^{p-1}(\wp)} \nabla_{a}^{\gamma} \wp,
\end{aligned}
$$

which is our claim.

Remark 2. In Theorem 7, if we take $\gamma=1$ then we get the following inequality

$$
\begin{aligned}
& \int_{m}^{\infty} k(\wp) v(\wp) w^{\rho}(\wp) g(\wp)\left(G^{\rho}(\wp)\right)^{-\urcorner_{1}} \mho^{p}(\wp) \nabla \wp \\
& \geqslant\left(\frac{p+\urcorner_{2}}{1-\urcorner_{1}+\vartheta}\right)^{p} \int_{m}^{\infty} \frac{k(\wp) v(\wp) w^{\rho}(\wp) r^{p}(\wp) \mho^{p}(\wp)\left(G^{\rho}(\wp)\right)^{p-\urcorner_{1}}}{g^{p-1}(\wp)} \nabla \wp .
\end{aligned}
$$

Corollary 5. Putting $\mathbb{T}=\mathbb{R}$ in (24) we obtain

$$
\begin{aligned}
& \int_{m}^{\infty} k(\wp) v(\wp) w(\wp) g(\wp)(G(\wp))^{\gamma-\urcorner_{1}+1} \mho^{p-\gamma+1}(\wp)(\wp-a)^{\gamma-1} d t \\
& \geqslant\left(\frac{p+\urcorner_{2}-\gamma+1}{\gamma-\urcorner_{1}+\vartheta}\right)^{p} \int_{m}^{\infty} \frac{k(\wp) v(\wp) w(\wp) r^{p}(\wp) \mho^{p}(\wp)(G(\wp))^{p-T_{1}+\gamma-1} \mho^{1-\gamma}(\wp)(\wp-a)^{\gamma-1} d t}{g^{p-1}(\wp)} d t, \\
& \text { where } \\
& G(\wp)=\int_{m}^{\wp} g(\omega)(\omega-a)^{\gamma-1} d \omega \text { with } G(\infty)=\infty, \text { and } \mho \mho(\wp)=\int_{\wp}^{\infty} r(\omega) \mho(\omega)(\infty-a)^{\gamma-1} d \omega \text {. }
\end{aligned}
$$

Corollary 6. Putting $\mathbb{T}=h \mathbb{Z}, h>0$ in (24) we obtain 


$$
\begin{aligned}
& \sum_{\wp=m}^{\infty} k(\wp) v(\wp) w(\wp-h) g(\wp) G^{\gamma-\urcorner_{1}-1}(\wp-h) \mho^{p-\gamma+1}(\wp)\left(\rho^{\gamma-1}(\wp)-a\right)_{h}^{(\gamma-1)} \\
& \geqslant\left(\frac{p+\rceil_{2}-\gamma+1}{\gamma-\rceil_{1}+\vartheta}\right)^{p} \sum_{\wp=m}^{\infty} \frac{k(\wp) v(\wp) w(\wp-h) r^{p}(\wp) \mho^{p}(\wp)(G(\wp-h))^{p-\urcorner_{1}+\gamma-1} \mho^{1-\gamma}(\wp-h)\left(\rho^{\gamma-1}(\wp)-a\right)_{h}^{(\gamma-1)}}{g^{p-1}(\wp)} . \\
& \text { where }
\end{aligned}
$$

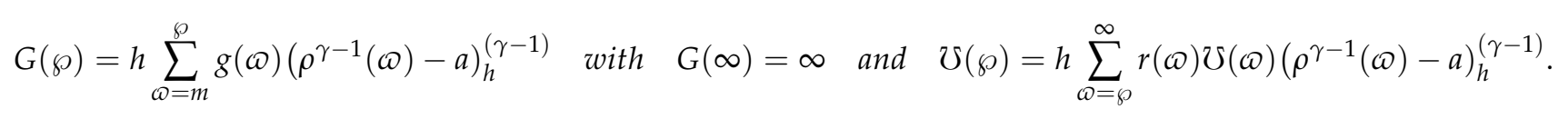

$$
\begin{aligned}
& \sum_{\wp=m}^{\infty} k(\wp) v(\wp) w(\wp-1) g(\wp) G^{\gamma-\urcorner_{1}-1}(\wp-1) \mho^{p-\gamma+1}(\wp)\left(\rho^{\gamma-1}(\wp)-a\right)^{(\gamma-1)} \\
& \geqslant\left(\frac{p+\rceil_{2}-\gamma+1}{\gamma-\urcorner_{1}+\vartheta}\right)^{p} \sum_{\wp=m}^{\infty} \frac{k(\wp) v(\wp) w(\wp-1) r^{p}(\wp) \mho^{p}(\wp)(G(\wp-1))^{p-\urcorner_{1}+\gamma-1} \mho^{1-\gamma}(\wp-1)\left(\rho^{\gamma-1}(\wp)-a\right)^{(\gamma-1)}}{g^{p-1}(\wp)} . \\
& \text { where } \\
& G(\wp)=\sum_{\omega=m}^{\wp} g(\infty)\left(\rho^{\gamma-1}(\varpi)-a\right)^{(\gamma-1)} \quad \text { with } \quad G(\infty)=\infty \quad \text { and } \quad \mho(\wp)=\sum_{\omega=\wp}^{\infty} r(\infty) \mho(\infty)\left(\rho^{\gamma-1}(\varpi)-a\right)^{(\gamma-1)} \text {. }
\end{aligned}
$$

Corollary 8. For $\mathbb{T}=q^{\mathbb{N}_{0}}$, in (24) we get

$$
\begin{aligned}
& \sum_{\wp=m}^{\infty} \wp\left(\rho^{\gamma-1}(\wp)-a\right)_{\tilde{q}}^{(\gamma-1)} k(\wp) v(\wp) w^{\rho}(\wp) g(\wp)\left(G^{\rho}(\wp)\right)^{\gamma-\urcorner_{1}-1} \mho^{p-\gamma+1}(\wp) \\
& \geqslant\left(\frac{p+\urcorner_{2}-\gamma+1}{\gamma-\rceil_{1}+\vartheta}\right)^{p} \sum_{\wp=m}^{\infty} \frac{\wp\left(\rho^{\gamma-1}(\wp)-a\right)_{\tilde{q}}^{(\gamma-1)} k(\wp) v(\wp) w^{\rho}(\wp) r^{p}(\wp) \mho \mho^{p}(\wp)\left(G^{\rho}(\wp)\right)^{p-\urcorner_{1}+\gamma-1} \mho^{1-\gamma}(\rho(\wp))}{g^{p-1}(\wp)} .
\end{aligned}
$$

where

$$
G(\wp)=(\tilde{q}-1) \sum_{\omega=m}^{\wp} \mathscr{\omega}\left(\rho^{\gamma-1}(\mathcal{\omega})-a\right)_{\tilde{q}}^{(\gamma-1)} g(\infty) \text { with } \quad G(\infty)=\infty . \mho(\wp)=,
$$

and

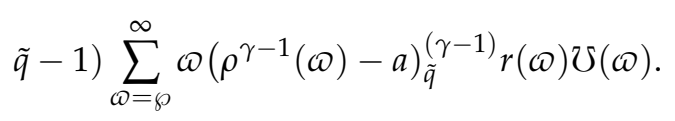

Theorem 8. Let $\mathbb{T}$ be a time scale with $m \in[0, \infty)_{\mathbb{T}}, \gamma \in(0,1]$ and $a>\wp$. Furthermore, assume the $r d$-continuous functions $v, g, r, \mho, k, w \geq 0$, on $[m, \infty)_{\mathbb{T}}$ with $k$ nondecreasing. Further, suppose we have $\vartheta,\urcorner_{2} \geq 0$ with $\frac{\nabla_{a}^{\gamma} w(\wp)}{w(\wp)} \geqslant \vartheta\left(\frac{\nabla_{a}^{\gamma} H(\wp)}{H^{\rho}(\wp)}\right)$ and $\left.\frac{\nabla_{a}^{\gamma} v(\wp)}{v^{\rho}(\wp)} \geqslant\right\rceil_{2}\left(\frac{\nabla_{a}^{\gamma} K(\wp)}{K(\wp)}\right)$, where

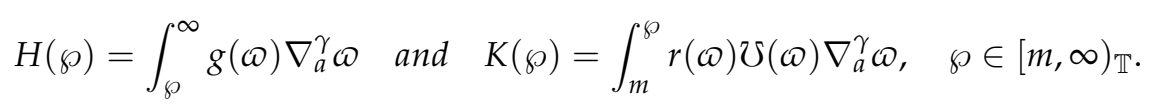

If $0<p<\gamma, 0 \leq\urcorner_{1}<\gamma$, then

$$
\begin{aligned}
& \int_{m}^{\infty} k^{\rho}(\wp) v^{\rho}(\wp) w(\wp) g(\wp) H^{\gamma-\urcorner_{1}-1}(\wp)\left(K^{\rho}(\wp)\right)^{p-\gamma+1} \nabla_{a}^{\gamma} \wp \\
& \geqslant\left(\frac{p-\gamma+\urcorner_{2}+1}{\gamma-\urcorner_{1}+\vartheta}\right)^{p} \int_{a}^{\infty} \frac{k^{\rho}(\wp) v^{\rho}(\wp) w(\wp) r^{p}(\wp) \mho^{p}(\wp) H^{p-\urcorner_{1}+\gamma-1}(\wp)\left(K^{\rho}(\wp)\right)^{(1-\gamma)}}{g^{p-1}(\wp)} \nabla_{a}^{\gamma} \wp .
\end{aligned}
$$


Proof. Applying (15) with

$$
\begin{aligned}
& \nabla_{a}^{\gamma} u(\wp)=w(\wp) g(\wp) H^{\gamma-\urcorner_{1}-1}(\wp) \quad \text { and } \quad z^{\rho}(\wp)=k^{\sigma}(\wp) v^{\rho}(\wp)\left(K^{\rho}(\wp)\right)^{p-\gamma+1} \text {, } \\
& \text { we have } \\
& \int_{m}^{\infty} k^{\rho}(\wp) v^{\rho}(\wp) w(\wp) g(\wp) H^{\gamma-\urcorner_{1}-1}(\wp)\left(K^{\rho}(\wp)\right)^{p-\gamma+1} \nabla_{a}^{\gamma} \wp \\
& =\left[u(\wp) k(\wp) v(\wp) K^{p-\urcorner_{1}+1}(\wp)\right]_{m}^{\infty}+\int_{m}^{\infty}(-u(\wp)) \nabla_{a}^{\gamma}\left(k(\wp) v(\wp) K^{p-\gamma+1}(\wp)\right) \nabla_{a}^{\gamma} \wp, \\
& u(\wp)=-\int_{\wp}^{\infty} w(\omega) g(\omega) H^{\gamma-\urcorner_{1}-1}(\omega) \nabla_{a}^{\gamma} \omega .
\end{aligned}
$$

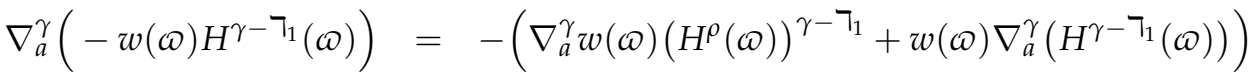

$$
\begin{aligned}
& \left.\leqslant-\left(\vartheta w(\omega)\left(H^{\rho}(\mathfrak{\omega})\right)^{\gamma-\urcorner_{1}-1} \nabla_{a}^{\gamma} H(\mathfrak{\omega})+(\gamma-\urcorner_{1}\right) w(\omega) H^{\gamma-\urcorner_{1}-1}(c) \nabla_{a}^{\gamma} H(\mathfrak{\omega})\right) .
\end{aligned}
$$

Because $\nabla_{a}^{\gamma} H(\omega)=-g(\omega) \leq 0, c \leqslant \omega$ and $\left.0 \leq\right\rceil_{1}<\gamma$, we get

$$
\begin{aligned}
\nabla_{a}^{\gamma}\left(-w(\omega) H^{\gamma-\urcorner_{1}}(\boldsymbol{\omega})\right) & \left.\leqslant \vartheta w(\omega) g(\omega) H^{\gamma-\urcorner_{1}-1}(\omega)+(\gamma-\urcorner_{1}\right) w(\omega) g(\omega) H^{\gamma-\urcorner_{1}-1}(\omega) \\
& \left.=(\gamma-\urcorner_{1}+\vartheta\right) w(\omega) g(\omega) H^{\gamma-\urcorner_{1}-1}(\omega) .
\end{aligned}
$$

Thus

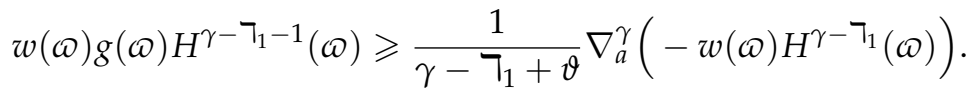

Hence

$$
\begin{aligned}
-u(\wp)=\int_{\wp}^{\infty} w(\omega) g(\omega) H^{\gamma-\urcorner_{1}-1}(\omega) \nabla_{a}^{\gamma} \omega & \geqslant \frac{1}{\gamma-\urcorner_{1}+\vartheta} \int_{\wp}^{\infty} \nabla_{a}^{\gamma}\left(-w(\omega) H^{\gamma-\urcorner_{1}}(\omega)\right) \nabla_{a}^{\gamma} \omega \\
& =\frac{1}{\gamma-\urcorner_{1}+\vartheta} w(\wp) H^{\gamma-\urcorner_{1}}(\wp) .
\end{aligned}
$$

Applying (14) and (16), we have $c \in[\rho(\wp)$, $]$ with

$$
\begin{aligned}
& \nabla_{a}^{\gamma}\left(k(\wp) v(\wp) K^{p-\gamma+1}(\wp)\right)=\nabla_{a}^{\gamma}(k(\wp) v(\wp)) K^{p-\gamma+1}(\wp)+k^{\rho}(\wp) v^{\rho}(\wp) \nabla_{a}^{\gamma}\left(K^{p-\gamma+1}(\wp)\right) \\
& =\nabla_{a}^{\gamma} k(\wp) v(\wp) K^{p-\gamma+1}(\wp)+k^{\rho}(\wp) \nabla_{a}^{\gamma} v(\wp) K^{p-\gamma+1}(\wp) \\
& +(p-\gamma+1) k^{\rho}(\wp) v^{\rho}(\wp) K^{p-\gamma}(c) \nabla_{a}^{\gamma} K(\wp) \text {. } \\
& \left.\nabla_{a}^{\gamma}\left(k(\wp) v(\wp) K^{p-\gamma+1}(\wp)\right) \geqslant\right\urcorner_{2} k^{\rho}(\wp) v^{\rho}(\wp) r(\wp) \mho(\wp) K^{p-\gamma}(\wp)+(p-\gamma+1) k^{\rho}(\wp) v^{\rho}(\wp) r(\wp) \mho(\wp) K^{p-\gamma}(\wp) \\
& \geqslant\left(p-\gamma+T_{2}+1\right) k^{\rho}(\wp) v^{\rho}(\wp) r(\wp) \mho(\wp) K^{p-\gamma}(\wp) . \\
& \int_{m}^{\infty} k^{\rho}(\wp) v^{\rho}(\wp) w(\wp) g(\wp) H^{\gamma-\urcorner_{1}-1}(\wp)\left(K^{\rho}(\wp)\right)^{p-\gamma+1} \nabla_{a}^{\gamma} \wp \\
& \geqslant \frac{p-\gamma+\rceil_{2}+1}{\gamma-\urcorner_{1}+\vartheta} \int_{m}^{\infty} k^{\rho}(\wp) v^{\rho}(\wp) w(\wp) r(\wp) \mho(\wp) H^{\gamma-\urcorner_{1}}(\wp) K^{p-\gamma}(\wp) \nabla_{a}^{\gamma} \wp .
\end{aligned}
$$


The last inequality can be rewritten as

$$
\begin{aligned}
& \int_{m}^{\infty} k^{\rho}(\wp) v^{\rho}(\wp) w(\wp) g(\wp) H^{\gamma-\urcorner_{1}+1}(\wp)\left(K^{\rho}(\wp)\right)^{p-\gamma+1} \nabla_{a}^{\gamma} \wp \\
& \geqslant \frac{p-\gamma+\urcorner_{2}+1}{\gamma-\urcorner_{1}+\vartheta} \int_{m}^{\infty}\left(\left(k^{\rho}(\wp) v^{\rho}(\wp) w(\wp) g(\wp)\right)^{(p-1) / p} H^{\left.(\gamma-\urcorner_{1}-1\right)(p-1) / p}(\wp) K^{(p-1)(p-\gamma+1) / p}(\wp)\right) \\
& \times\left(\frac{\left(k^{\rho}(\wp) v^{\rho}(\wp) w(\wp)\right)^{1 / p} r(\wp) \mho(\wp) H^{\left.(p-\urcorner_{1}+\gamma-1\right) / p}(\wp) K^{(1-\gamma) / p}(\wp)}{g^{(p-1) / p}(\wp)}\right) \nabla_{a}^{\gamma} \wp .
\end{aligned}
$$

Applying (11) with the index $p$ and the index $p /(p-1)$, gets

$$
\begin{aligned}
& \int_{a}^{\infty} k^{\rho}(\wp) v^{\rho}(\wp) w(\wp) g(\wp) H^{\gamma-\urcorner_{1}-1}(\wp)\left(K^{\rho}(\wp)\right)^{p-\rho+1} \nabla_{a}^{\gamma} \wp \\
& \geqslant \frac{p-\gamma+\rceil_{2}+1}{\gamma-\rceil_{1}+\vartheta}\left(\int_{m}^{\infty} k^{\rho}(\wp) v^{\rho}(\wp) w(\wp) g(\wp) H^{\gamma-\urcorner_{1}-1}(\wp) K^{p-\gamma+1}(\wp) \nabla_{a}^{\gamma} \wp\right)^{(p-1) / p} \\
& \quad \times\left(\int_{m}^{\infty} \frac{k^{\rho}(\wp) v^{\rho}(\wp) w(\wp) r^{p}(\wp) \mho^{p}(\wp) H^{p-\urcorner_{1}+\gamma-1}(\wp) K^{1-\gamma}(\wp)}{g^{p-1}(\wp)} \nabla_{a}^{\gamma} \wp\right)^{1 / p} .
\end{aligned}
$$

This implies that

$$
\begin{aligned}
& \int_{m}^{\infty} k^{\rho}(\wp) v^{\rho}(\wp) w(\wp) g(\wp) H^{\gamma-\urcorner_{1}-1}(\wp)\left(K^{\rho}(\wp)\right)^{p-\gamma+1} \nabla_{a}^{\gamma} \wp \\
& \geqslant\left(\frac{p-\gamma+\rceil_{2}+1}{\gamma-\rceil_{1}+\vartheta}\right)^{p} \int_{a}^{\infty} \frac{k^{\rho}(\wp) v^{\rho}(\wp) w(\wp) r^{p}(\wp) \mho^{p}(\wp) H^{p-\urcorner_{1}+\gamma-1}(\wp) K^{1-\gamma}(\wp)}{g^{p-1}(\wp)} \nabla_{a}^{\gamma} \wp .
\end{aligned}
$$

This concludes our result.

Remark 3. Putting $\gamma=1$ in our result Theorem 8, then we get the following inequality

$$
\begin{aligned}
& \int_{m}^{\infty} k^{\rho}(\wp) v^{\rho}(\wp) w(\wp) g(\wp)(H(\wp))^{-\urcorner_{1}}\left(K^{\rho}(\wp)\right)^{p} \nabla \wp \\
& \geqslant\left(\frac{p+\rceil_{2}}{1-\rceil_{1}+\vartheta}\right)^{p} \int_{m}^{\infty} \frac{k^{\rho}(\wp) v^{\rho}(\wp) w(\wp) r^{p}(\wp) \mho^{p}(\wp) H^{p-\urcorner_{1}}(\wp)}{g^{p-1}(\wp)} \nabla \wp .
\end{aligned}
$$

Corollary 9. Putting $\mathbb{T}=\mathbb{R}$ in (28) gets

$$
\begin{aligned}
& \int_{m}^{\infty} k(\wp) v(\wp) w(\wp) g(\wp) H^{\gamma-\urcorner_{1}-1}\left(K^{\rho}(\wp)\right)^{p-\gamma+1}(\wp)(\wp-a)^{\gamma-1} d t \\
& \geqslant\left(\frac{p+\urcorner_{2}-\gamma+1}{\gamma-\urcorner_{1}+\vartheta}\right)^{p} \int_{m}^{\infty} \frac{k(\wp) v(\wp) w(\wp) r^{p}(\wp) \mho^{p}(\wp) H^{p-\urcorner_{1}+\gamma-1}(\wp) K^{1-\gamma}(\wp)(\wp-a)^{\gamma-1} d t}{g^{p-1}(\wp)} d t
\end{aligned}
$$

where

$$
H(\wp)=\int_{m}^{\infty} g(\omega)(\omega-a)^{\gamma-1} d \omega \quad \text { and } \quad K(\wp)=\int_{m}^{\wp} r(\omega) \mho(\omega)(\omega-a)^{\gamma-1} d \omega .
$$

Corollary 10. Putting $\mathbb{T}=h \mathbb{Z}, h>0$ in (28) gets

$$
\begin{aligned}
& \sum_{\wp=m}^{\infty} k(\wp-h) v(\wp-h) w(\wp) g(\wp) H^{\gamma-\urcorner_{1}-1}(\wp) K^{p-\gamma+1}(\wp-h)\left(\rho^{\gamma-1}(\wp)-a\right)_{h}^{(\gamma-1)} \\
& \geqslant\left(\frac{p+\rceil_{2}-\gamma+1}{\gamma-\urcorner_{1}+\vartheta}\right)^{p} \sum_{\wp=m}^{\infty} \frac{k(\wp-h) v(\wp-h) w(\wp) r^{p}(\wp) \mho^{p}(\wp) H^{p-\urcorner_{1}+\gamma-1}(\wp) K^{1-\gamma}(\wp)\left(\rho^{\gamma-1}(\wp)-a\right)_{h}^{(\gamma-1)}}{g^{p-1}(\wp)} .
\end{aligned}
$$

where

$H(\wp)=h \sum_{\omega=\wp}^{\infty} g(\omega)\left(\rho^{\gamma-1}(\omega)-a\right)_{h}^{(\gamma-1)}$ with and $K(\wp)=h \sum_{\omega=m}^{\wp} r(\omega) \mho(\omega)\left(\rho^{\gamma-1}(\omega)-a\right)_{h}^{(\gamma-1)}$. 
Corollary 11. If $\mathbb{T}=\mathbb{Z}$, with $h=1$ in our result Theorem 10 then, (28) obtains to

$$
\begin{aligned}
& \sum_{\wp=m}^{\infty} k(\wp-1) v(\wp-1) w(\wp) g(\wp) H^{\gamma-\urcorner_{1}-1}(\wp) K^{p-\gamma+1}(\wp-1)\left(\rho^{\gamma-1}(\wp)-a\right)^{(\gamma-1)} \\
& \geqslant\left(\frac{p+\urcorner_{2}-\gamma+1}{\gamma-\urcorner_{1}+\vartheta}\right)^{p} \sum_{\wp=m}^{\infty} \frac{k(\wp-1) v(\wp-1) w(\wp) r^{p}(\wp) \mho^{p}(\wp) H^{p-\urcorner_{1}+\gamma-1}(\wp) K^{1-\gamma}(\wp)\left(\rho^{\gamma-1}(\wp)-a\right)(\gamma-1)}{g^{p-1}(\wp)} .
\end{aligned}
$$

where

$H(\wp)=\sum_{\omega=\wp}^{\infty} g(\omega)\left(\rho^{\gamma-1}(\omega)-a\right)^{(\gamma-1)}$ with and $K(\wp)=h \sum_{\omega=m}^{\wp} r(\omega) \mho \delta(\omega)\left(\rho^{\gamma-1}(\omega)-a\right)^{(\gamma-1)}$.

Corollary 12. Putting $\mathbb{T}=q^{\mathbb{N}_{0}}$, in (28) we obtains

$$
\begin{gathered}
\sum_{\wp=m}^{\infty} \wp\left(\rho^{\gamma-1}(\wp)-a\right)_{\tilde{q}}^{(\gamma-1)} k^{\rho}(\wp) v^{\rho}(\wp) w(\wp) g(\wp) H^{\gamma-\urcorner_{1}-1}\left(K^{\rho}(\wp)\right)^{p-\gamma+1} \\
\geqslant\left(\frac{p+\urcorner_{2}-\gamma+1}{\gamma-\urcorner_{1}+\vartheta}\right)^{p} \sum_{\wp=m}^{\infty} \frac{\wp\left(\rho^{\gamma-1}(\wp)-a\right)_{\tilde{q}}^{(\gamma-1)} k^{\rho}(\wp) v^{\rho}(\wp) w(\wp) r^{p}(\wp) \mho^{p}(\wp) H^{p-\urcorner_{1}+\gamma-1}(\wp) K^{1-\gamma}(\wp)}{g^{p-1}(\wp)} . \\
H(\wp)=(\tilde{q}-1) \sum_{\omega=\wp}^{\infty} \omega\left(\rho^{\gamma-1}(\omega)-a\right)_{\tilde{q}}^{(\gamma-1)} g(\omega) \text { and } K(\wp)=(\tilde{q}-1) \sum_{\omega=m}^{\wp} \omega\left(\rho^{\gamma-1}(\omega)-a\right)_{\tilde{q}}^{(\gamma-1)} r(\omega) \mho \delta(\omega) .
\end{gathered}
$$

Theorem 9. Assume $\mathbb{T}$ is a time scale with $m \in[0, \infty)_{\mathbb{T}}, \gamma \in(0,1]$ and $\wp>a$. In addition, let the $r d$-continuous functions $g_{\|} v, r, \mho, k, w \geq 0$, on $[m, \infty)_{\mathbb{T}}$ with $k$ nonincreasing. Moreover, suppose there exist $\vartheta, \mathrm{T}_{2} \geq 0$ such that $\frac{\nabla_{a}^{\gamma} w(\wp)}{w^{\rho}(\wp)} \leqslant \vartheta\left(\frac{H(\wp)}{H(\wp)}\right)$ and $\left.\frac{\nabla_{a}^{\gamma} v(\wp)}{v(\wp)} \leqslant\right\rceil_{2}\left(\frac{\nabla_{a}^{\gamma} \mho(\wp)}{v^{\rho}(\wp)}\right)$, where

$$
\begin{aligned}
& w(m)=0, \quad H(\wp)=\int_{\wp}^{\infty} g(\omega) \nabla_{a}^{\gamma} \omega \text { and } \quad \mho(\wp)=\int_{\wp}^{\infty} r(\omega) \mho(\omega) \nabla_{a}^{\gamma} \omega, \quad \wp \in[m, \infty)_{\mathbb{T}} . \\
& \text { If } 0<p<\gamma \text { and }\rceil_{1}>\vartheta+1 \text {, then }
\end{aligned}
$$$$
\begin{aligned}
& \int_{m}^{\infty} k(\wp) v(\wp) w^{\rho}(\wp) g(\wp) H^{\gamma-\urcorner_{1}-1}(\wp) \mho^{p-\gamma+1}(\wp) \nabla_{a}^{\gamma} \wp \\
& \geqslant\left(\frac{p+\urcorner_{2}-\gamma+1}{\urcorner_{1}-\vartheta-\gamma}\right)^{p} \int_{m}^{\infty} \frac{k(\wp) v(\wp) w^{\rho}(\wp) r^{p}(\wp) \mho^{p}(\wp) H^{\left.(1-\gamma+\urcorner_{1}\right)(p-1)}(\wp)\left(\zeta^{\rho}(\wp)\right)^{(1-\gamma)}}{g^{p-1}(\wp)\left(H^{\rho}(\wp)\right)^{\left.p(\urcorner_{1}-\gamma\right)}} \nabla_{a}^{\gamma} \wp .
\end{aligned}
$$

Proof. Applying (15), obtains

$$
\begin{aligned}
& \int_{m}^{\infty} k(\wp) v(\wp) w^{\rho}(\wp) g(\wp) H^{\gamma-\urcorner_{1}-1}(\wp) \mho^{p-\gamma+1}(\wp) \nabla_{a}^{\gamma} \wp \\
& =\left[u(\wp) k(\wp) v(\wp) \mho^{p-\gamma+1}(\wp)\right]_{m}^{\infty}+\int_{m}^{\infty} u^{\rho}(\wp) \nabla_{a}^{\gamma}\left(-k(\wp) v(\wp) \mho^{p-\gamma+1}(\wp)\right) \nabla_{a}^{\gamma} \wp, \\
& u(\wp)=\int_{m}^{\wp} w^{\rho}(\omega) g(\omega) H^{\gamma-\urcorner_{1}-1}(\omega) \nabla_{a}^{\gamma} \omega . \\
& \nabla_{a}^{\gamma}\left(w(\omega) H^{\gamma-\urcorner_{1}}(\boldsymbol{\omega})\right)=\nabla_{a}^{\gamma} w(\omega) H^{\gamma-\urcorner_{1}}(\omega)+w^{\rho}(\omega) \nabla_{a}^{\gamma}\left(H^{\gamma-\urcorner_{1}}(\boldsymbol{\omega})\right) \\
& \left.\leqslant \vartheta w^{\rho}(\omega) \nabla_{a}^{\gamma} H(\omega) H^{\gamma-\urcorner_{1}-1}(\omega)+(\gamma-\urcorner_{1}\right) w^{\rho}(\omega) H^{\gamma-\urcorner_{1}-1}(c) \nabla_{a}^{\gamma} H(\omega) .
\end{aligned}
$$


Since $\nabla_{a}^{\gamma} H(\omega)=-g(\omega) \leq 0, c \leqslant \omega$ and $\rceil_{1}>1$, we get

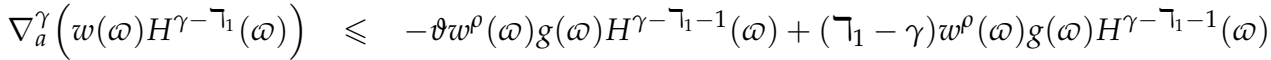

$$
\begin{aligned}
& \left.=(\urcorner_{1}-\vartheta-\gamma\right) w^{\rho}(\omega) g(\omega) H^{\gamma-\urcorner_{1}-1}(\omega) \text {. }
\end{aligned}
$$

This gives us that

$$
w^{\rho}(\boldsymbol{\omega}) g(\boldsymbol{\omega}) H^{\gamma-\urcorner_{1}-1}(\boldsymbol{\omega}) \geqslant \frac{1}{\urcorner_{1}-\vartheta-\gamma} \nabla_{a}^{\gamma}\left(w(\omega) H^{\gamma-\urcorner_{1}}(\boldsymbol{\omega})\right) .
$$

Therefore

$$
\begin{aligned}
u^{\rho}(\wp)=\int_{m}^{\rho(\wp)} w^{\rho}(\omega) g(\omega) H^{\gamma-\urcorner_{1}-1}(\omega) \nabla_{a}^{\gamma} \omega & \geqslant \frac{1}{\urcorner_{1}-\vartheta-\gamma} \int_{m}^{\rho(\wp)} \nabla_{a}^{\gamma}\left(w(\omega) H^{\gamma-\urcorner_{1}}(\omega)\right) \nabla_{a}^{\gamma} \omega \\
& =\frac{1}{\urcorner_{1}-\vartheta-\gamma}\left(w^{\rho}(\wp)\left(H^{\rho}(\wp)\right)^{\gamma-\urcorner_{1}}-w(m) H^{\gamma-\urcorner_{1}}(m)\right) \\
& \geqslant \frac{1}{\urcorner_{1}-\vartheta-\gamma} w^{\rho}(\wp)\left(H^{\rho}(\wp)\right)^{\gamma-\urcorner_{1}} .
\end{aligned}
$$

Employing (14) and (16), one have $c \in[\rho(\wp)$, $\wp]$ with

$$
\begin{aligned}
& \nabla_{a}^{\gamma}\left(-k(\wp) v(\wp) \mho^{p-\gamma+1}(\wp)\right)=-\left(\nabla_{a}^{\gamma}(k(\wp) v(\wp))\left(\mho^{\rho}(\wp)\right)^{p-\rho+1}+k(\wp) v(\wp) \nabla_{a}^{\gamma}\left(\mho^{p-\rho+1}(\wp)\right)\right) \\
& =-\left(\nabla_{a}^{\gamma} k(\wp) v^{\rho}(\wp)\left(\zeta^{\rho}(\wp)\right)^{p-\gamma+1}+k(\wp) \nabla_{a}^{\gamma} v(\wp)\left(\zeta^{\rho}(\wp)\right)^{p-\gamma+1}\right. \\
& \left.+(p-\gamma+1) k(\wp) v(\wp) \mho^{p-\gamma}(c) \nabla_{a}^{\gamma} \mho \delta(\wp)\right) . \\
& \left.\geqslant(p+\rceil_{2}+\gamma-1\right) k(\wp) v(\wp) r(\wp) \mho(\wp)\left(\mho^{\rho}(\wp)\right)^{p-\gamma} . \\
& \int_{m}^{\infty} k(\wp) v(\wp) w^{\rho}(\wp) g(\wp) H^{\gamma-\urcorner_{1}-1}(\wp) \mho^{p-\gamma+1}(\wp) \nabla_{a}^{\gamma} \wp \\
& \geqslant \frac{p+\rceil_{2}+\gamma-1}{\urcorner_{1}-\vartheta-\gamma} \int_{m}^{\infty} k(\wp) v(\wp) w^{\rho}(\wp) r(\wp) \mho(\wp)\left(H^{\rho}(\wp)\right)^{\gamma-\urcorner_{1}}\left(\mho^{\rho}(\wp)\right)^{p-\gamma} \nabla_{a}^{\gamma} \wp, \\
& \int_{m}^{\infty} k(\wp) v(\wp) w^{\rho}(\wp) g(\wp) H^{\gamma-\urcorner_{1}-1}(\wp) \mho^{p-\gamma+1}(\wp) \nabla_{a}^{\gamma} \wp \\
& \geqslant \frac{p+\urcorner_{2}+\gamma-1}{\urcorner_{1}-\vartheta-\gamma} \int_{m}^{\infty}\left(\left(k(\wp) v(\wp) w^{\rho}(\wp) g(\wp)\right)^{(p-1) / p} H^{\left.(\gamma-\urcorner_{1}-1\right)(p-1) / p}(\wp)\left(\mho^{\rho}(\wp)\right)^{(p-\gamma+1)(p-1) / p}\right) \\
& \times\left(\frac{\left(k(\wp) v(\wp) w^{\rho}(\wp)\right)^{1 / p} r(\wp) \mho(\wp) H^{\left.(1-\gamma+\urcorner_{1}\right)(p-1) / p}(\wp)\left(\mho^{\rho}(\wp)\right)^{(1-\gamma) / p}}{g^{(p-1) / p}(\wp)\left(H^{\rho}(\wp)\right)^{\urcorner_{1}-\gamma}}\right) \nabla_{a}^{\gamma} \wp .
\end{aligned}
$$


Applying the dynamic Hölder inequality (11) with the index $p$ and the index $p /(p-1)$, obtains

$$
\begin{aligned}
& \int_{m}^{\infty} k(\wp) v(\wp) w^{\rho}(\wp) g(\wp) H^{\gamma-\rceil_{1}-1}(\wp) \mho^{p-\gamma+1}(\wp) \nabla_{a}^{\gamma} \wp \\
& \geqslant \frac{p+\rceil_{2}-\gamma+1}{\rceil_{1}-\vartheta-\gamma}\left(\int_{m}^{\infty} k(\wp) v(\wp) w^{\rho}(\wp) g(\wp) H^{\gamma-\urcorner_{1}-1}(\wp)\left(\mho^{\rho}(\wp)\right)^{p-\gamma+1} \nabla_{a}^{\gamma} \wp\right)^{(p-1) / p} \\
& \times\left(\int_{m}^{\infty} \frac{k(\wp) v(\wp) w^{\rho}(\wp) r^{p}(\wp) \mho \mho^{p}(\wp) H^{\left.(1-\gamma+\urcorner_{1}\right)(p-1)}(\wp)\left(\mho^{\rho}(\wp)\right)^{(1-\gamma)}}{g^{p-1}(\wp)\left(H^{\rho}(\wp)\right)^{p\left(7_{1}-\gamma\right)}} \nabla_{a}^{\gamma} \wp\right)^{1 / p},
\end{aligned}
$$

which implies

$$
\begin{aligned}
& \int_{m}^{\infty} k(\wp) v(\wp) w^{\rho}(\wp) g(\wp) H^{\gamma-\urcorner_{1}-1}(\wp) \mho^{p-\gamma+1}(\wp) \nabla_{a}^{\gamma} \wp \\
& \geqslant\left(\frac{p+\rceil_{2}-\gamma+1}{\urcorner_{1}-\vartheta-\gamma}\right)^{p} \int_{m}^{\infty} \frac{k(\wp) v(\wp) w^{\rho}(\wp) r^{p}(\wp) \mho^{p}(\wp) H^{\left.(1-\gamma+\urcorner_{1}\right)(p-1)}(\wp)\left(\mho^{\rho}(\wp)\right)^{(1-\gamma)}}{g^{p-1}(\wp)\left(H^{\rho}(\wp)\right)^{\left.p(\urcorner_{1}-\gamma\right)}} \nabla_{a}^{\gamma} \wp,
\end{aligned}
$$

that is our required inequality.

Remark 4. In our result Theorem 9, putting $\gamma=1$ then we get the following inequality

$$
\begin{aligned}
& \int_{m}^{\infty} k(\wp) v(\wp) w^{\rho}(\wp) g(\wp) H^{-\urcorner_{1}}(\wp) \mho^{p}(\wp) \nabla \wp \\
& \geqslant\left(\frac{p+\urcorner_{2}}{\rceil_{1}-\vartheta-1}\right)^{p} \int_{m}^{\infty} \frac{k(\wp) v(\wp) w^{\rho}(\wp) r^{p}(\wp) \mho \mho^{p}(\wp) H^{\urcorner_{1}(p-1)}}{g^{p-1}(\wp)\left(H^{\rho}(\wp)\right)^{\left.p(\urcorner_{1}-1\right)}} \nabla \wp .
\end{aligned}
$$

Corollary 13. Putting $\mathbb{T}=\mathbb{R}$ in the result 9 , inequality (32) reduces to

$$
\begin{aligned}
& \int_{m}^{\infty} k(\wp) v(\wp) w(\wp) g(\wp) H^{\gamma-7_{1}-1}(\wp) \mho^{p-\gamma+1}(\wp)(\wp-a)^{\gamma-1} d t \\
& \geqslant\left(\frac{p+\rceil_{2}-\gamma+1}{\rceil_{1}-\vartheta-\gamma}\right)^{p} \int_{m}^{\infty} \frac{k(\wp) v(\wp) w(\wp) r^{p}(\wp) \mho^{p}(\wp) H^{p-\rceil_{1}+\gamma-1}(\wp) \mho^{1-\gamma}(\wp)}{g^{p-1}(\wp)}(\wp-a)^{\gamma-1} d t .
\end{aligned}
$$

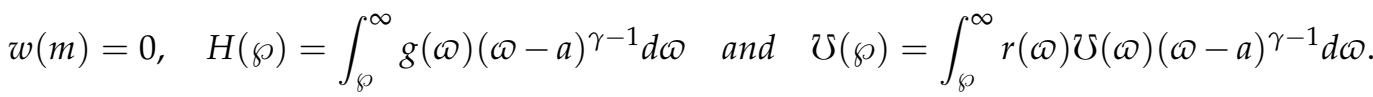

Corollary 14 . For $\mathbb{T}=h \mathbb{Z}, h>0$ in the result 9 , inequality (32) reduces to

$$
\begin{aligned}
& \sum_{\wp=m}^{\infty} k(\wp) v(\wp) w(\wp-h) g(\wp) H^{\gamma-\urcorner_{1}-1}(\wp) \mho^{p-\gamma+1}(\wp-h)\left(\rho^{\gamma-1}(\wp)-a\right)_{h}^{(\gamma-1)} \\
& \geqslant\left(\frac{p+\rceil_{2}-\gamma+1}{\urcorner_{1}-\vartheta-\gamma}\right)^{p} \sum_{\wp=m}^{\infty} \frac{k(\wp) v(\wp) w(\wp-h) r^{p}(\wp) \mho^{p}(\wp) H^{\left.(1-\gamma+\rceil_{1}\right)(p-1)}(\wp) \mho^{1-\gamma}(\wp-h)\left(\rho^{\gamma-1}(\wp)-a\right)_{h}^{(\gamma-1)}}{g^{p-1}(\wp)\left(H^{\rho}(\wp)\right)^{\left.p(\urcorner_{1}-\gamma\right)}} . \\
& \text { where } \\
& w(m)=0, H(\wp)=h \sum_{\omega=\wp}^{\infty} g(\mathcal{\omega})\left(\rho^{\gamma-1}(\boldsymbol{\omega})-a\right)_{h}^{(\gamma-1)} \text { and } \quad \mho(\wp)=h \sum_{\omega=\wp}^{\infty} r(\mathcal{\omega}) \mho(\mathcal{\omega})\left(\rho^{\gamma-1}(\mathcal{\omega})-a\right)_{h}^{(\gamma-1)} \text {. }
\end{aligned}
$$

Corollary 15. If $\mathbb{T}=\mathbb{Z}$, with $h=1$ in the result 14 then, (32) reduces to 


$$
\begin{aligned}
& \sum_{\wp=m}^{\infty} k(\wp) v(\wp) w(\wp-1) g(\wp) H^{\gamma-\urcorner_{1}-1}(\wp) \mho^{p-\gamma+1}(\wp-1)\left(\rho^{\gamma-1}(\wp)-a\right)^{(\gamma-1)}
\end{aligned}
$$

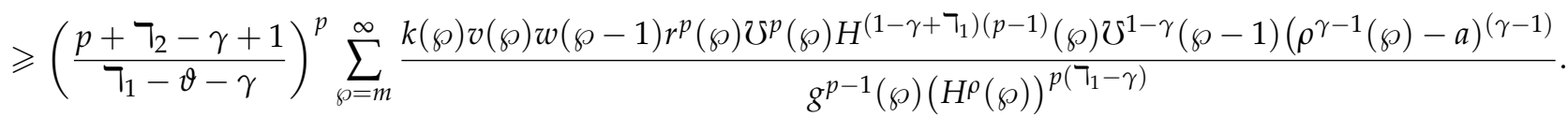

$$
\begin{aligned}
& \text { where } \\
& w(m)=0, H(\wp)=\sum_{\omega=\wp}^{\infty} g(\omega)\left(\rho^{\gamma-1}(\omega)-a\right)^{(\gamma-1)} \quad \text { and } \quad \mho \zeta(\wp)=\sum_{\omega=\wp}^{\infty} r(\omega) \mho \delta(\omega)\left(\rho^{\gamma-1}(\omega)-a\right)^{(\gamma-1)} \text {. } \\
& \text { Corollary 16. Putting } \mathbb{T}=q^{\mathbb{N}_{0}} \text {, in (32) we get } \\
& \sum_{\wp=m}^{\infty} \wp\left(\rho^{\gamma-1}(\wp)-a\right)_{\tilde{q}}^{(\gamma-1)} k(\wp) v(\wp) w^{\rho}(\wp) g(\wp) H^{\gamma-\tau_{1}-1}(\wp) \mho^{p-\gamma+1}(\wp) \\
& \geqslant\left(\frac{p+\rceil_{2}-\gamma+1}{\rceil_{1}-\vartheta-\gamma}\right)^{p} \sum_{\wp=m}^{\infty} \frac{\wp\left(\rho^{\gamma-1}(\wp)-a\right)_{\tilde{q}}^{(\gamma-1)} k(\wp) v(\wp) w^{\rho}(\wp) r^{p}(\wp) \mho^{p}(\wp) H^{\left.(1-\gamma+\rceil_{1}\right)(p-1)}(\wp)\left(\mho^{\rho}(\wp)\right)^{p-\gamma}}{g^{p-1}(\wp) H^{\left.p(\urcorner_{1}-\gamma\right)}(\wp)} . \\
& w(m)=0, \quad H(\wp)=(\tilde{q}-1) \sum_{\omega=\wp}^{\infty} \omega\left(\rho^{\gamma-1}(\omega)-a\right)_{\tilde{q}}^{(\gamma-1)} g(\omega) \quad \text { and } \quad \mho(\wp)=(\tilde{q}-1) \sum_{\omega=\wp}^{\infty} \omega\left(\rho^{\gamma-1}(\omega)-a\right)_{\tilde{q}}^{(\gamma-1)} r(\omega) \mho(\omega) .
\end{aligned}
$$

\section{Conclusions}

In this manuscript, by employing $\nabla$-integral fractional of order $\gamma \in(0,1]$, many $\nabla$ inequalities Hardy-type are introduced. For the sake of completeness, we applied the main results to some nonuniform time scales.

Author Contributions: Conceptualization, resources and methodology, A.A.E.-D. and S.D.M.; investigation, supervision, J.A.; data curation, S.S.A.; writing-original draft preparation, A.A.E.-D.; writing-review and editing, J.A.; project administration, A.A.E.-D. and S.D.M. All authors read and agreed to the published version of the manuscript.

Funding: This research received no external funding.

Institutional Review Board Statement: Not applicable.

Informed Consent Statement: Not applicable.

Data Availability Statement: Not applicable.

Acknowledgments: The authors extend their appreciation to the Research Supporting Project number (RSP-2022/167), King Saud University, Riyadh, Saudi Arabia.

Conflicts of Interest: The authors declare no conflict of interest.

\section{References}

1. Ozkan, U.M.; Yildirim, H. Hardy-Knopp-type inequalities on time scales. Dynam. Syst. Appl. 2008, 17, 477-486.

2. Saker, S.H.; O'Regan, D.; Agarwal, R.P. Dynamic inequalities of Hardy and Copson type on time scales. Analysis 2014, 34, 391-402. [CrossRef]

3. Oguntuase, J.A.; Persson, L.E. Time scales Hardy-type inequalities via superquadracity. Ann. Funct. Anal. 2014, 5, 61-73. [CrossRef]

4. Donchev, T.; Nosheen, A.; Pečarić, J. Hardy-type inequalities on time scale via convexity in several variables. ISRN Math. Anal. 2013, 2013, 903196. [CrossRef]

5. $\quad$ Agarwal, R.P.; O’Regan, D.; Saker, S.H. Hardy Type Inequalities on Time Scales; Springer: Cham, Switzerland, 2016 ; pp. x+305. [CrossRef]

6. Hardy, G.H. Note on a theorem of Hilbert. Math. Z. 1920, 6, 314-317. [CrossRef]

7. Hardy, G.H. Notes on some points in the integral calculus (LX). Messenger Math. 1925, 54, 150-156.

8. Copson, E.T. Note on Series of Positive Terms. J. Lond. Math. Soc. 1928, 3, 49-51. [CrossRef] 
9. Renaud, P.F. A reversed Hardy inequality. Bull. Austral. Math. Soc. 1986, 34, 225-232. [CrossRef]

10. El-Deeb, A.A.; Makharesh, S.D.; Baleanu, D. Dynamic Hilbert-Type Inequalities with Fenchel-Legendre Transform. Symmetry 2020, 12, 582. [CrossRef]

11. Bohner, M.; Peterson, A. Dynamic Equations on Time Scales; An Introduction with Applications; Birkhäuser Boston, Inc.: Boston, MA, USA, 2001; pp. x+358. [CrossRef]

12. Agarwal, R.P.; Bohner, M.; Peterson, A. Inequalities on time scales: A survey. Math. Inequal. Appl. 2001, 4, 535-557. [CrossRef]

13. Hilscher, R. A time scales version of a Wirtinger-type inequality and applications. J. Comput. Appl. Math. 2002, 141, 219-226. [CrossRef]

14. Řehák, P. Hardy inequality on time scales and its application to half-linear dynamic equations. J. Inequal. Appl. 2005, 2005, 495-507. [CrossRef]

15. Agarwal, R.P.; Mahmoud, R.R.; O’Regan, D.; Saker, S.H. Some reverse dynamic inequalities on time scales. Bull. Aust. Math. Soc. 2017, 96, 445-454. [CrossRef]

16. El-Deeb, A.A.; El-Sennary, H.A.; Khan, Z.A. Some reverse inequalities of Hardy type on time scales. Adv. Differ. Equ. 2020, 2020, 402. [CrossRef]

17. El-Deeb, A.A.; Elsennary, H.A.; Baleanu, D. Some new Hardy-type inequalities on time scales. Adv. Differ. Equ. 2020, $2020,441$. [CrossRef]

18. Saker, S.H.; Kenawy, M.; AlNemer, G.; Zakarya, M. Some fractional dynamic inequalities of Hardy's type via conformable calculus. Mathematics 2020, 8, 434. [CrossRef]

19. Zakaryaed, M.; Altanji, M.; AlNemer, G.; El-Hamid, A.; Hoda, A.; Cesarano, C.; Rezk, H.M. Fractional Reverse Coposn's Inequalities via Conformable Calculus on Time Scales. Symmetry 2021, 13, 542. [CrossRef]

20. El-Deeb, A.A.; Awrejcewicz, J. Novel Fractional Dynamic Hardy-Hilbert-Type Inequalities on Time Scales with Applications. Mathematics 2021, 9, 2964. [CrossRef]

21. Hilger, S. Analysis on measure chains-A unified approach to continuous and discrete calculus. Results Math. 1990, 18, 18-56. [CrossRef]

22. Bohner, M.; Peterson, A. (Eds.) Advances in Dynamic Equations on Time Scales; Birkhäuser Boston, Inc.: Boston, MA, USA, 2003; pp. xii+348. [CrossRef]

23. Rahmat, M.R.S.; Noorani, M.S.M. A new conformable nabla derivative and its application on arbitrary time scales. Adv. Differ. Equations 2021, 2021, 1-27.

24. Nwaeze, E.R.; Torres, D.F. Chain rules and inequalities for the BHT fractional calculus on arbitrary timescales. Arab. J. Math. 2017, 6, 13-20. [CrossRef]

25. Bendouma, B.; Hammoudi, A. A nabla conformable fractional calculus on time scales. Electron. J. Math. Anal. Appl. 2019, 7, 202-216.

26. Khalil, R.; Horani, M.A.; Yousef, A.; Sababheh, M. A new definition of fractional derivative. J. Comput. Appl. Math. 2014, 264, 65-70. [CrossRef] 